\title{
Middle East Respiratory Syndrome Coronavirus and the One Health concept
}

\author{
Maged Gomaa Hemida ${ }^{\text {Corresp. 1,2 }}$ \\ ${ }^{1}$ Department of Microbiology and Parasitology, College of Veterinary Medicine, King Faisal University, Al-Hufuf, Al-Hasa, Saudi Arabia \\ ${ }^{2}$ Department of Virology, faculty of veterinary medicine, Kafrelsheikh University, Egypt, Kafrelsheikh University, Kafrelsheikh, Kafrelsheikh, Egypt \\ Corresponding Author: Maged Gomaa Hemida \\ Email address: mhemida@kfu.edu.sa
}

Middle East Respiratory Syndrome Coronavirus (MERS-CoV) is one of the major threats to the healthcare systems in some countries, especially in the Arabian Peninsula. MERS-CoV is considered an ideal example of the One Health concept. This is due to the animals especially dromedary camels play important roles in the transmission and sustainability of the virus, and the virus can be transmitted through aerosols of infected patients into the environment. However, there is some debate regarding the origin of MERS-CoV either from bats or other unknown reservoirs. The dromedary camel is the only identified animal reservoir to date. These animals play important roles in sustaining the virus in certain communities and may act as an amplifier of the virus by secreting it in their body fluids, especially in nasal and rectal discharges. MERS-CoV has been detected in the nasal and rectal secretions of infected camels, and MERS-CoV of this origin has full capacity to infect human airway epithelium in both in vitro and in vivo models. Other evidence confirms the direct transmission of MERS-CoV from camels to humans, though the role of camel meat and milk products has yet to be well studied. Human-to-human transmission is well documented through contact with an active infected patient or some silently infected persons. Furthermore, there are some significant risk factors of individuals in close contact with a positive MERS-CoV patient, including sleeping in the same patient room, removing patient waste (urine, stool, and sputum), and touching respiratory secretions from the index case. Outbreaks within family clusters have been reported, whereby some blood relative patients were infected through their wives in the same house were not infected. Some predisposing genetic factors favor MERS-CoV infection in some patients, which is worth investigating in the near future. The presence of other comorbidities may be another factor. Overall, there are many unknown/confirmed aspects of the virus/human/animal network. Here, the most recent advances in this context are discussed, and the possible reasons behind the emergence and sustainability of MERS-COV in certain regions are presented. Identification of the exact mechanism of transmission of MERS-CoV from 
camels to humans and searching for new reservoir/s are of high priority. This will reduce the shedding of the virus into the environment, and thus the risk of human infection can be mitigated. 


\section{Maged Gomaa Hemida ${ }^{1,2 *}$}

5

$6 \quad{ }^{1}$ Department of Microbiology and Parasitology, College of Veterinary Medicine, King Faisal

7 University, Al-Hufuf, Al-Hasa, Saudi Arabia

8 2Department of Virology, faculty of veterinary medicine, Kafrelsheikh University, Egypt,

9 Kafrelsheikh University, Kafrelsheikh, Kafrelsheikh, Egypt

11 Corresponding author*

12

13

14 *Maged Hemida: mhemida@kfu.edu.sa; Tel.: +966-53027-0662

15

17 Running title: MERS-CoV/human/animal interaction 
24 Abstract:

25 Middle East Respiratory Syndrome Coronavirus (MERS-CoV) is one of the major threats to the

26 healthcare systems in some countries, especially in the Arabian Peninsula. MERS-CoV is

27 considered an ideal example of the One Health concept. This is due to the animals especially

28 dromedary camels play important roles in the transmission and sustainability of the virus, and the

29 virus can be transmitted through aerosols of infected patients into the environment. However,

30 there is some debate regarding the origin of MERS-CoV either from bats or other unknown

31 reservoirs. The dromedary camel is the only identified animal reservoir to date. These animals

32 play important roles in sustaining the virus in certain communities and may act as an amplifier of

33 the virus by secreting it in their body fluids, especially in nasal and rectal discharges. MERS-

$34 \mathrm{CoV}$ has been detected in the nasal and rectal secretions of infected camels, and MERS-CoV of

35 this origin has full capacity to infect human airway epithelium in both in vitro and in vivo

36 models. Other evidence confirms the direct transmission of MERS-CoV from camels to humans,

37 though the role of camel meat and milk products has yet to be well studied. Human-to-human

38 transmission is well documented through contact with an active infected patient or some silently

39 infected persons. Furthermore, there are some significant risk factors of individuals in close

40 contact with a positive MERS-CoV patient, including sleeping in the same patient room,

41 removing patient waste (urine, stool, and sputum), and touching respiratory secretions from the 
42 index case. Outbreaks within family clusters have been reported, whereby some blood relative

43 patients were infected through their wives in the same house were not infected. Some

44 predisposing genetic factors favor MERS-CoV infection in some patients, which is worth

45 investigating in the near future. The presence of other comorbidities may be another factor.

46 Overall, there are many unknown/confirmed aspects of the virus/human/animal network. Here,

47 the most recent advances in this context are discussed, and the possible reasons behind the

48 emergence and sustainability of MERS-CoV in certain regions are presented. Identification of

49 the exact mechanism of transmission of MERS-CoV from camels to humans and searching for

50 new reservoir/s are of high priority. This will reduce the shedding of the virus into the

51 environment, and thus the risk of human infection can be mitigated.

\section{1. Introduction}

53 The main reason behind developing this article is to summarize the current understanding about

54 MERS-CoV in the context of the One Health concept. In this article, I highlight the known

55 information about the MERS-CoV infection and its pathogenesis in humans, the patterns of

56 MERS-CoV in dromedary camels, the potential roles of other animals in the transmission cycle

57 of MERS-CoV, and the interaction of MERS-CoV/humans/animals. I elaborate on how some

58 strategies can be used to stop or reduce the frequencies of MERS-CoV outbreaks based on the

59 One Health concept, identified some gaps in the literature, and drew conclusions. The one health

60 concept established to ensure the good health and well beings of the human, animal and the 
61 environment. The human health is mainly affected by the environment health as well as the

62 animal health (Lerner \& Berg 2015).

63

64 SURVEY METHODOLOGY

65 For this review article, I conducted a literature search of the most up-to-date published articles on

66 MERS-CoV in the past 7 years. First, I focused the introduction section on the historical

67 background of coronaviruses and the One Health concept. Then, I highlighted the most up-to-

68 date literature from PubMed central, Google Scholar and ResearchGate on the interaction of

69 MERS-CoV/humans/animals. I identified some important gaps in the research dealing with

70 MERS-CoV/human/environment in the context of the One Health concept. I also summarized the

71 current acceptable theories on the emergence and evolution of MERS-CoV. Finally, I highlighted

72 progress to date in the control of MERS-CoV. Historically, MERS-CoV was first identified in

73 Saudi Arabia in a patient suffered from severe pneumonia and shortening of breath. The virus

74 was called the novel coronavirus at that time (Zaki et al. 2012). Another retrospective study

75 conducted in Jordan early 2012 revealed the detection of this novel coronavirus in 11 patients.

76 Eight out of them were from the health care workers (Hijawi et al. 2013).

2. Coronaviruses: the past, present, and future

Coronaviruses are a large group of viruses causing many health problems (respiratory, enteric, and nervous syndromes) in various species of animals and humans. Six human coronaviruses that have been identified to date (HCoV-229E, HCoV-OC43, HCoV-NL-63, 
81 HCoV-HUK-1, SARS-CoV, and MERS-CoV). Two out of them emerged in the past 15 years

82 (Lau \& Chan 2015), namely, the severe acute respiratory syndrome coronavirus (SARS-CoV)

83 and the Middle East Respiratory Syndrome Coronavirus (MERS-CoV). SARS-CoV emerged in

842003 in China and spread to many countries throughout the world (Peiris et al. 2003).

85 Approximately 8000 people were infected, and 10\% of them died (Aronin \& Sadigh 2004). Only

869 years later, MERS-CoV emerged in Saudi Arabia (Zaki et al. 2012). This is a relatively short

87 period for the emergence of a new coronavirus. One of the main reasons behind the rapid

88 emergence of new coronaviruses is the poor proofreading capability of their RNA polymerases

89 (Hofer 2013). This is in addition to the possibility of the recombination of different

90 coronaviruses (Makino et al. 1986), and it will not be surprising if new coronaviruses emerge in

91 the near future. MERS-CoV continues to pose great challenges to the healthcare system of some

92 countries in the Middle East and Arabian Peninsula. Since its discovery late in 2012 (Zaki et al.

93 2012), there are ongoing reports to the World Health Organization (WHO) from some countries

94 in the Middle East, especially the Arabian Peninsula, with spread to other countries around the

95 globe. According to the latest WHO statistics, there have been a total of 2,428 laboratory-

96 confirmed cases of MERS-CoV infection including at least 838 deaths (reported case fatality rate

97 of $35.0 \%$ ) (WHO 2018). The continuous ongoing reports on MERS-CoV suggesting the

98 presence of some factors favor its sustainability in certain regions. There are many uncertain

99 aspects of the virus evolution, pathogenesis, and transmission cycle. Unfortunately, recently,

100 there was some decline in the rate of research on the virus from different aspects (Hemida et al.

101 2017b). This hampered the production of new data about the MERS-CoV from different aspects.

102 Below, I summarize the current understanding of the virus in the context of the One Health

103 concept. 


\section{3. Coronaviruses and the One Health concept}

105 The One Health concept is an interesting concept outlining the close interaction among humans,

106 animals and the environment (Destoumieux-Garzon et al. 2018). Currently, there are two

107 coronaviruses candidates representing the One Health Concept, SARS-CoV and MERS-CoV.

108 Animals play important roles in the transmission cycle of both viruses (Alshukairi et al. 2018;

109 Wang et al. 2005). Both viruses were proven to be of zoonotic origin (Gao et al. 2016). The palm

110 civet cat played important role in the transmission cycle of SARS-CoV (Wang et al. 2005). Some

111 patients proved to visit one restaurant serving the civet cats as a meal (Wang et al. 2005). Culling

112 of the civet cats resulted in marked decline in the reported SARS-CoV cases and now become

113 extinct. Many studies made a direct link between the exposure to camel and its meat and milk

114 products and MERS-CoV human cases (Reusken et al. 2014). Several studies reported the

115 presence of MERS-CoV specific antibodies in sera of human came in close contact with camels

116 (Reusken et al. 2014; Reusken et al. 2016). Meanwhile, MERS-CoV was isolated from air pf

117 positive dromedary camel herds in Saudi Arabia (Azhar et al. 2014).

\section{4. MERS-CoV in Humans}

119 MERS-CoV may infect a wide group of people ranging from very young ages, even infants

120 less than one year of age, to 109 years of age (CDC 2016). However, children are less likely to

121 be infected with MERS-CoV when compared to adults, and if infected, they tend to have

122 asymptomatic or mild disease (Arwady et al. 2016). The reason for this is still not entirely clear

123 and requires further study. 
124 The case fatality rate is always very high in case of the immunocompromised infected

125 patients especially those who are suffering from chronic diseases such as cancer, diabetes, blood

126 pressure, kidney problems, etc. (Arwady et al. 2016).

Human-to-human transmission is reported in many cases. MERS-CoV replicates efficiently

128 in various in vitro and ex vivo models (Chan et al. 2014). Moreover, many family clusters and

129 hospital outbreaks were reported in the past 5 years (Arwady et al. 2016; Drosten et al. 2014;

130 Memish et al. 2013). This confirms the potential spread of MERS-CoV among those who are in

131 close contact in the population (Mollers et al. 2015). The most at-risk groups are healthcare

132 workers including nurses, medical doctors and other hospital staff and the elderly with

133 underlying chronic diseases (Arabi et al. 2014).

134 The prevalence rate of MERS-CoV in primary cases among males is relatively higher than

135 that of females (Darling et al. 2017), which may be because exposure to infected dromedary

136 camels is much higher in males than in females.

MERS-CoV infection triggers some unique interferons and cytokine gene expression

138 profiles. The virus seems to be a poor interferon inducer (Chan et al. 2014). This suggests the

139 potential immune evasion strategies triggered by the virus to hijack the host immune system and

140 may be responsible for the high fatality rate, at least in part. Viral spreading among people seems

141 to not yet be very efficient. Those in close contact are among the at-risk groups for infection

142 (Drosten et al. 2014), as observed in many hospital outbreaks as well as family clusters (Alfaraj

143 et al. 2018; Choi et al. 2017; Xiao et al. 2018). This suggests that transmission of the virus

144 among people requires exposure to a high viral load, which will sometimes produce active

145 infection in people who are in close contact. Several MERS-CoV family clusters have been 
146 reported (Drosten et al. 2014). Interestingly, MERS-CoV is reported in the dromedary camels in

147 many African countries (Egypt, Nigeria, Tunisia, and Ethiopia), but no primary human cases

148 have been reported in these countries to date (Ali et al. 2017; Roess et al. 2016; van Doremalen

149 et al. 2017), which may be related to some variation in the circulating Asian and African strains 150 of MERS-CoV.

151 Some important deletions in the MERS-CoV currently circulating in dromedary camels from

152 Africa were recently reported (Chu et al. 2018). These deletions may explain at least in part the

153 reason behind the variations in the pathogenesis among the Asian and African strains of MERS-

$154 \mathrm{CoV}$. Another potential reason behind the absence of human cases in the African countries is the

155 diverse cultural habits among people in Africa and the Arabian Peninsula (FAO 2016). People in

156 Arabian Peninsula get in more close touch with camels during the camel show, sports, trade than

157 in Africa. This make the human risk of exposure much higher in the AP than Africa. MERS-CoV

158 infection varies from severe respiratory illness accompanied by a high fever and respiratory

159 distress to mild asymptomatic cases. Patients are usually admitted to the intensive care unit

160 (ICU) and provided with a source of oxygen. Most cases result in pneumonia, which is fatal in

161 almost $40 \%$ of the affected patients (Hong et al. 2017; Rubio et al. 2018). Some patients may

162 develop renal failure [13]. Several MERS-CoV travel-associated infections were in many cases

163 associated with the Middle East (Bayrakdar et al. 2015; Rubio et al. 2018). Among these

164 reported was the Korean outbreak in early 2015 (Choi et al. 2017; Kim et al. 2017; Xiao et al.

165 2018). One Korean citizen visited some countries in the Middle East and then returned home ill.

166 This person visited several healthcare facilities in Korea. This resulted in the largest MERS-CoV

167 human outbreak outside the Arabian Peninsula (AP) (Xiao et al. 2018). This outbreak confirmed

168 the human-to-human transmission. During this outbreak, MERS-CoV was isolated from air 
169 samples from the hallways of the healthcare facilities close to the hospitalized patients (Xiao et

170 al. 2018). This at least explains in part the rapid development of MERS-CoV hospital outbreaks.

\section{5. MERS-CoV in animals}

172 Since the discovery of MERS-CoV late in 2012 (Zaki et al. 2012), many research groups

173 have searched for its potential animal reservoir/s. Dromedary camels are the only currently

174 proven reservoir for MERS-CoV (Hemida et al. 2014; Hemida et al. 2017b; Reusken et al. 2014;

175 Reusken et al. 2016). Interestingly, others were able to trace the virus back 30 years ago in

176 dromedary camel specimens in retrospective studies (Corman et al. 2014; Hemida et al. 2014;

177 Reusken et al. 2014). All these data suggest that the virus has been circulating for decades

178 without being recognized. Although the actual and typical clinical features of the MERS-CoV

179 natural infection in dromedary camels is not well documented to date, very few studies reported

180 these patterns under experimental infection approaches (Adney et al. 2014). Based on these

181 findings, camels do not show any pathognomonic signs despite a subtle fever and mild nasal

182 discharge for up to 6 days post-infection (dpi) (Hemida et al. 2014). Meanwhile, shedding of the

183 infectious virus was reported in the experimentally infected camels started at 2 dpi up to the 7 th

184 dpi (Adney et al. 2014). Interestingly, viral RNA was still detected at 35 dpi (Adney et al. 2014),

185 though it is not known whether the viral RNAs may act as potential sources of infection similar

186 to some other positive-sense RNA viruses. No viral shedding in the oral secretions, rectal swabs,

187 urine, or sera of these animals has been reported (Adney et al. 2014), in contrast to the detection

188 of the virus in the fecal specimens and swabs under natural viral field infection (Hemida et al.

189 2014). These finding suggesting differential patterns of MERS-CoV infection between natural

190 and experimental approaches. Further studies are required to understand the natural MERS-CoV

191 infection in dromedary camels, which may be achieved by conducting long-term longitudinal 
192 studies as well as careful monitoring of the virus infection in large populations of camels. On

193 necropsy examination of MERS-CoV, experimentally infected dromedary camels revealed only

194 mild-to-moderate inflammatory reactions in the upper respiratory tract (Khalafalla et al. 2015).

195 Detection of the viral antigens in the tissue sections of the turbinate bone and the upper

196 respiratory tract was reported (Adney et al. 2014). Interestingly, seroconversion of the inoculated

197 animals was reported to begin at 14 dpi (Hemida et al. 2014), indicating that MERS-CoV

198 induces a robust humoral immune response after infection. More recently, one longitudinal study

199 reported the possibility of MERS-CoV infection in seropositive animals. This raises concern

200 about the role of the antibodies in the protection of the MERS-CoV infection (Hemida et al.

201 2017a). It seems that all the members of the family Camelidae (dromedary, alpaca, and llamas)

202 are susceptible to MERS-CoV infection (Corman et al. 2014; Vergara-Alert et al. 2017). David

203 et al. (2018) reported the presence of antibodies against MERS-CoV in some alpacas and llamas

204 in Israel but only used commercial ELISA kits, and they did not address the possibility of cross-

205 reactivity with other coronaviruses especially $\mathrm{BCoV}$. It had been previously showed that there is

206 clear cross-reactivity between MERS-CoV and BCoV in dromedary camels (David et al. 2018).

207 Interestingly, one study showed an absence of any detectable antibodies of MERS-CoV in the

208 sera of Bactrian camels (Chan et al. 2015), though this is the only study to report this finding

209 concerning the seronegativity of Bactrian camels to MERS-CoV. It is not well known whether

210 the absence of detectable MERS-CoV antibodies in the sera of Bactrians camels is due to the

211 geographical location of the tested animals in Mongolia, far from the Middle East and Africa.

212 This may be supported by similar findings in dromedary camels in Australia and the Canary

213 Islands (Crameri et al. 2015). Another possibility is that this might be due to some genetic

214 factors, which contribute to the resistance of Bactrians to MERS-CoV infections; this point is 
215 worthy of further investigation. Experimental MERS-CoV infection in both alpacas and llamas

216 showed a similar pattern to that of dromedary camels (Crameri et al. 2016; Vergara-Alert et al.

217 2017), which suggested that both animals might act as a model animal for the study of MERS-

$218 \mathrm{CoV}$ in vivo. However, the experimental infection of pigs with MERS-CoV did not reveal as

219 much infection as that reported in alpacas and llamas (Vergara-Alert et al. 2017). Active MERS-

$220 \mathrm{CoV}$ particles were neither retrieved from the experimentally infected animals nor from the close

221 contact non-infected animals during the duration of this study (Vergara-Alert et al. 2017). This

222 result suggested that pigs might not play an active role in the transmission of MERS-CoV.

223 Although bats are considered the main reservoir for many coronaviruses, their roles in the

224 MERS-CoV still need further clarifications. One study reported the presence of MERS-CoV in

225 one specimen collected from bats in Saudi Arabia (Memish et al. 2013). The genome sequence

226 of this particular virus showed almost 100\% identity to a MERS-CoV index case (Memish et al.

227 2013). More recently, Jamaican fruit bats were found to be permissible for MERS-CoV infection

228 (Munster et al. 2016). However, MERS-CoV-infected bats did not show any apparent clinical

229 signs; however, viral shedding was reported in the swabs from bats up to 9 dpi. The clinical

230 profiles and viral shedding curve during the course of the MERS-CoV infection in these bats

231 look similar to that of dromedary camels (Munster et al. 2016), yet the amount of infectious viral

232 shedding in bats is less compared to that in dromedary camels. This species of bat is not the most

233 relevant for MERS-CoV infection, but this study offers some insights into the molecular

234 pathogenesis of MERS-CoV in bats. Interestingly, another study revealed the expression of

235 MERS-CoV receptors (dipeptidyl peptidase-4, DPP4) in the respiratory and digestive tracts of

236 some insectivorous bats (Widagdo et al. 2017). An interesting study tested the potential roles of

237 other species in the transmission of MERS-CoV such as cattle, sheep, goats, donkeys, buffaloes, 
238 mules, and horses from Egypt, Tunisia and Senegal (Kandeil et al. 2019). This study revealed the

239 presence of neutralizing antibodies in the sera of some sheep and goats. Meanwhile, viral RNA

240 was detected in swabs collected from some sheep, goats and donkeys from these countries

241 (Kandeil et al. 2019). Several attempts were made to identify an appropriate experimental animal

242 model for MERS-CoV. The Syrian hamster was non-permissive to MERS-CoV infection (de Wit

243 et al. 2013). Experimental infection of this animal neither develops any clinical signs or

244 pathology nor produces any cytokines after infection (de Wit et al. 2013). This was in contrast to

245 New Zealand white rabbits, which showed active infection after inoculation with the MERS-

246 CoV (Monchatre-Leroy et al. 2017). Furthermore, both rhesus macaques and common

247 marmosets supported MERS-CoV infection (Yu et al. 2017). Additionally, both the transgenic

248 and the transduced mice expressing the dipeptidyl peptidase 4 human receptors worked as a

249 model for MERS-CoV studies (Zhao et al. 2015). Interestingly, a new study reported the

250 seropositivity of some sheep and goat to MERS-CoV from Tunisia, Senegal and Egypt (Kandeil

251 et al. 2019). Same study reported the detection of the viral RNAs in samples from cow, sheep,

252 goat and donkeys from Egypt (Kandeil et al. 2019). This highlights the importance of continuous

253 surveillance and searching for new reservoir/s for the MERS-CoV transmission cycle.

\section{6. MERS/human/animal interaction}

It is now well accepted that human exposure to MERS-CoV-infected dromedary camels is a 256 predisposing factor to human infection, particularly in immunocompromised people (Zumla et al. 2015). Based on the latest WHO reports, the prognosis of MERS-CoV infection is poor for elderly patients who have chronic diseases such as cancer, diabetes, kidney failure, etc. (Arabi et al. 2014). Transmission of MERS-CoV from dromedary camels to humans has been proven

260 indirectly in some previous reports (Azhar et al. 2014). One study showed strong evidence of 
261 direct transmission of MERS-CoV from an infected camel to its owner, which was confirmed by

262 comparing the viral genome sequencing of the virus isolated from the infected dromedary camel

263 to that isolated from its owner. Both viruses were almost a 100\% match (Azhar et al. 2014).

264 Meanwhile, this study reported the detection of MERS-CoV nucleic acid in air samples from the

265 indicated dromedary camel barn during the active course of the viral infection (Azhar et al.

266 2014).

267 There is a debate about the role of the dromedary camel's milk and meat products and by-

268 products in the transmission of MERS-CoV. Experimental introduction of MERS-CoV to raw

269 milk revealed little difference between the virus stock in milk and that kept in DMEM (van

270 Doremalen et al. 2014). Due to their culture, some people in the Middle East would drink raw

271 camel milk in efforts to seek treatment for some diseases such as diabetes. The authors

272 acknowledge that MERS-CoV was introduced into the dromedary camel milk at a high dose and

273 that the viral RNA was detected in a limited concentration in the camel's milk (van Doremalen et

274 al. 2017). Thus, drinking the raw camel milk poses a great risk to the people consuming this milk

275 without any heat treatment or pasteurization (van Doremalen et al. 2014; Zhou et al. 2017). One

276 study connected the infection of some people to the drinking of the milk from one infected camel

277 (Memish et al. 2015). However, another study was conducted in Qatar to assess the possibility of

278 acquiring the infection from contaminated teats and udders of infected she-camels during the

279 process of milking (Reusken et al. 2014), though no active MERS-CoV shedding in milk has yet

280 to be reported. Further studies are encouraged to come to a conclusion about the potential role of

281 raw camel milk in the transmission of MERS-CoV. Nonetheless, the role of camel meat in the

282 transmission of MERS-CoV has not been studied carefully to date. Thus, special attention should

283 be paid to the efficient cooking of camel meat and its products as well as thorough boiling of 
284 camel milk. It is suggested that people not drink raw camel milk to avoid any risk of infection

285 not only with MERS-CoV but also with other pathogens such as Brucellosis (Garcell et al.

286 2016). Some studies reported that MERS-CoV is one of the occupational zoonotic viral diseases,

287 as was claimed based on some studies investigating the seroconversion of some at-risk group of

288 people to MERS-CoV. This study reported the presence of specific MERS-CoV antibodies in

289 approximately $3 \%$ of the workers in some slaughterhouses in Qatar (Farag et al. 2015). On the

290 other hand, some studies reported the absence of any detectable antibodies in the sera of some

291 herdsmen, veterinarians, and slaughterhouses in Saudi Arabia (Hemida et al. 2015). One possible

292 explanation for the variations between the two studies is the difference in the sensitivity of the

293 techniques used. Those two studies used two different techniques to report the presence/absence

294 of the MERS-CoV antibodies in the sera of at-risk people (Farag et al. 2015; Hemida et al.

295 2015). Regardless, further investigation on a large-scale basis is required to solidify this

296 conclusion about MERS-CoV.

297 7. Gaps on the MERS-CoV-related research

There is more to be known about the molecular biology of MERS-CoV. Identification of the

DPP-4 as viral receptors does not rule out the presence of other co-receptors or

300

transcription/translation factors that favor the virus infection in a certain host. There are many

301 immune evasion strategies triggered by MERS-CoV to hijack the host immune responses, and

302 the mechanisms of such strategies have not been well studied. Moreover, there are many

303 unknown aspects especially in the context of the MERS-CoV/human/animal interaction.

304 Meanwhile, some studies were conducted on a small scale or with low numbers of

305 animals/specimens and reported some important conclusion. These studies need further

306 confirmation, and refinement of some of these observations is urgently needed in the near future.

Peer] reviewing PDF | (2018:12:33627:2:2:NEW 21 Jun 2019) 
307 Here, we highlight some gaps in the research regarding the evolution and transmission of MERS-

308 CoV. Presumably, there might be an unidentified reservoir in the transmission cycle of MERS-

$309 \mathrm{CoV}$. Although respiratory infection still is the main route of MERS-CoV infection, the exact

310 mechanism of transmission of MERS-CoV from dromedary camels to humans is still not well

311 understood. The possibility of another reservoir in the transmission cycle of MERS-CoV has not

312 been ruled out. Thus, there might be a missing link in the chain of the human/camel interaction.

313 Meanwhile, the exact modes of transmission of MERS-CoV from dromedary camels to humans

314 have not been well clarified, and the typical pattern of the natural MERS-CoV infection in

315 dromedary camels has not been well studied. Additionally, the potential role of most camel

316 secretions and excretions has not been fully understood. The seroprevalence of MERS-CoV was

317 reported in the dromedary camels from different countries in Africa and Asia (Ali et al. 2017;

318 Hemida et al. 2014), though feral camels in Australia and the Canary islands were found to be

319 seronegative (Crameri et al. 2015). The reason behind this phenomenon may be due to these

320 regions being isolated lands and away from the MENA region as described above; it may also be

321 due to the absence of an active camel movement between the Middle East and Africa and these

322 regions of the world. Very few studies reported the cross-reactivity between MERS-CoV and

323 other coronaviruses such as the bovine coronavirus $(\mathrm{BCoV})$ that might infect dromedary camels.

324 It is unknown if this might be the reason behind the high seroprevalence of MERS-CoV among

325 the dromedary camel population. This may be due to the high frequency of exposure to the

326 MERS-CoV infection during the camel's life, the cross-reactivity of other coronaviruses, or an

327 unknown mechanism related to the dromedary camel's immune system. These considerations

328 require further studies. There is ongoing demand for the development of novel diagnostic assays

329 for coronaviruses, and special interest should be paid to those techniques that enable the 
330 simultaneous detection of the viral nucleic acids and those that can simultaneously distinguish

331 between the antibodies for several coronaviruses. Furthermore, it is not well understood why

332 only the Bactrian camels among the family Camelidae did not seroconvert to MERS-CoV

333 infection (Chan et al. 2015). The genetic susceptibility of certain human populations, especially

334 blood-related people, is not clear in the context of MERS-CoV infection. There are several levels

335 of human exposure to dromedary camels, such as camel attendants, workers in camel abattoirs,

336 veterinarians inspecting their carcasses, and camel owners. Those groups of people are in close

337 contact with camels for various amounts of time and are considered to be a high-risk group of

338 people due to the long time they spend in close contact with the dromedary camels. Meanwhile,

339 there is an urgent need to develop a risk scoring system for human exposure to the dromedary

340 camels.

341 8. Current theories in the MERS-CoV/human/animals interaction

342 It is believed that there is some unidentified reservoirs in the context of MERS-CoV

343 transmission presenting the virus to the community. This virus is able to infect dromedary

344 camels, which act as an amplifier host for the virus, favoring the circulation of the virus in some

345 camel herds. The virus has the ability to circulate among the animals in the same herd and the

346 camel herds in close proximity to them (Figure 1). MERS-CoV in camels has the full potential to

347 infect the human especially immunocompromised persons. Once the virus infects a human, there

348 is always a possibility of infecting other people, especially closely-related individuals (figure 1),

349 including household relatives and workers plus healthcare workers such as doctors and nurses.

350 Infection depends on the level of exposure to the infected person, and MERS-CoV infection in

351 humans ranges from very severe cases of pneumonia to death. Currently available data indicate

352 that severely infected individuals can shed the infectious virus into the environment (Kim et al. 
353 2016), though there are few data regarding the capacity of mildly infected individuals to transmit

354 the virus. Asymptomatic individuals, however, are unlikely to transmit the virus (Moon \& Son 355 2017).

\section{Potential reasons for the emergence and the spread of MERS-CoV}

There are many factors behind the emergence, sustainability and spread of MERS-CoV. The presence of an unidentified MERS-CoV reservoir in the transmission cycle is still considered, and this unknown reservoir may contribute substantially to the suitability of the virus in certain regions. Dromedary camels remain the amplifier of the virus; the close contact of these animals to the human population in certain regions of Africa and Asia may pose a great risk for human infection and indirectly contribute to the spread of the virus. Additionally, public animal markets, especially for dromedary camels, may act as an amplifier of the virus. This poses a great risk to

364 the surrounding community. Interesting study addressed the mapping of MERS-CoV cases in association with the environmental conditions and camel exposure (Reeves et al. 2015). This study revealed that camel exposure is a key predisposing factor for some of MERS-CoV human cases (Reeves et al. 2015). The lack of active surveillance programs for respiratory viruses, especially coronaviruses, may result in many subclinical or mild cases of MERS-CoV being missed in a certain population. These patients may shed the virus in their secretions and may act as a source of infection to other persons in close contact with them. Although many MERS-CoV

371 vaccine and drug candidates are being researched, none are available yet. All these factors may

372 favor the sustainability of MERS-CoV in certain regions.

\section{Current progress on the control of MERS-CoV}


374 Interestingly, the case fatality rate of MERS-CoV among the affected population dropped from

375 almost 50\% in 2012 to $34 \%$ early 2019 (WHO 2018), and we may relate this progress in the

376 control of MERS-CoV over the past 7 years to many factors. First, identification of the main

377 reservoir of the virus, namely, the dromedary camel (Hemida et al. 2014). Second, continuous

378 molecular and serological surveillance of MERS-CoV among the dromedary camel population in

379 the Arabian Peninsula and Africa (Corman et al. 2014; Farag et al. 2015; Hemida et al. 2017a;

380 Hemida et al. 2017b; Khalafalla et al. 2015; Reusken et al. 2014). Currently, testing the

381 population of camels in regional camel markets is associated with shutting down of the market in

382 case of positive shedding of MERS-CoV by the animals. I believe this will substantially

383 minimize the risk of community-acquired infections through these positive populations. Third,

384 vaccination of dromedary camels, especially animals under two years of age, will have a great

385 impact on the reduction of the viral shedding from these animals to the surrounding community.

386 This will also have a great positive impact on the reduction of the number of reported human

387 infections. Fourth, there has been progress in the current understanding of viral tropism,

388 pathogenesis, and mode of transmission in the past five years (Chan et al. 2014; Widagdo et al.

389 2017). Fifth, new strategies have been adopted to reduce the spread of infection in health care

390 units (Rajakaruna et al. 2017). Sixth, some therapeutic and control approaches for MERS-CoV

391 such as cyclosporine, ribavirin and interferon show promising trends for the treatment of MERS-

392 CoV-infected patients (Al-Tawfiq et al. 2014; de Wilde et al. 2013). Meanwhile, good progress

393 has been made in screening large numbers of drugs/therapies for the treatment of MERS-CoV

394 (Han et al. 2018; He et al. 2019; Niu et al. 2018; Totura \& Bavari 2019). This may lead to the

395 development of some effective novel drugs against MERS-CoV infection in the near future.

396 11. One Health-based interventions to stop MERS-CoV outbreaks 
397 To stop MERS-CoV outbreaks, there are several strategies to be adopted in the context of the

398 One Health concept. Some strategies are related to the animal, while others are related to human

399 health. The main objective is to minimize or stop the viral shedding from dromedary camels to

400 the environment (Figure 2). This may be achieved in many ways including regular monitoring of

401 the population of dromedary camels. Active animal shedders need to be identified, and

402 quarantine measures should applied until they stop shedding the virus. Vaccination of young

403 dromedary camel calves should occur during their first 6 months of life, which will minimize the

404 chances of these animals becoming infected and actively passing the virus to older animals and

405 then to the environment. Reorganization and reshaping of the camel industry includes allocating

406 the camel markets away from the cities. Global awareness concerning the necessity of thorough

407 boiling and cooking of the camel milk and meat products, respectively, should take place.

408 Animal abattoirs should be established far away from large cities. They should not use mixed-

409 animal platforms, and each platform should deal with one species of animal. Thorough

410 decontamination of animals' biological wastes in abattoirs should occur using the appropriate

411 standard protocols. Regular surveillance of MERS-CoV among the population especially during

412 the active peak of virus shedding by the animals should occur during November to April every

413 year. People who are in close contact with the camels should wear proper personal protective

414 equipment at all times.

\section{12. Conclusions}

416 At almost 7 years after its emergence, there are ongoing reports of MERS-CoV infection from

417 time to time. This may be related to many unknown aspects of the viral evolution and pathogenesis.

418 Some of these unknown aspects are the following. (1) Little is still known about virus/host

419 interactions and how MERS-CoV hijacks the host immune system. (2) Potential reservoirs in the 
420 context of the MERS-CoV/human/animal network need to be identified. (3) Why do MERS-CoV-

421 infected dromedary camels not exhibit obvious clinical signs during active viral shedding? (4)

422 Does the presence of neutralizing antibodies in the sera of animals protect them against future

423 active infection by the virus? (5) Does vaccination of dromedary camels have a positive impact on

424 controlling the spread of MERS-CoV among dromedary camels? More research is urgently needed

425 to explore the unknown aspects of the MERS-CoV/human/animal network.

\section{Figure legends}

428

429

430

431

432

433

434

435

436

437

438

439

440

441

442
Figure 1: Current acceptable theories in the MERS-CoV/human/animals interaction

There might be an unknown reservoir in the transmission cycle of MERS-CoV. Bats play a role in the context of MERS-CoV transmission. The virus is transmitted to dromedary camels through an unknown mechanism. The dromedary camels act as amplifying hosts for the virus. MERS-CoV is transmitted from dromedary camels to humans through the respiratory aerosols and some other unknown mechanisms. The virus is then transmitted among the human population through respiratory routes. The human-to-human transmission has been confirmed. The humanto-camel transmission still needs further clarification. Question marks indicate the non-confirmed phenomenon.

Figure 2. Some interventions based on the One Health-based to stop MERS-CoV outbreaks

\section{References}

Adney DR, van Doremalen N, Brown VR, Bushmaker T, Scott D, de Wit E, Bowen RA, and Munster VJ. 2014. Replication and shedding of MERS-CoV in upper respiratory tract of inoculated dromedary camels. Emerg Infect Dis 20:1999-2005. 10.3201/eid2012.141280

PeerJ reviewing PDF | (2018:12:33627:2:2:NEW 21 Jun 2019) 
443

444

445

446

447

448

449

450

451

452

453

454

455

456

457

458

459

460

461

462

463

464

465

466

467

468

469

470

471

472

473

474

475

476

477

478

479

Al-Tawfiq JA, Momattin H, Dib J, and Memish ZA. 2014. Ribavirin and interferon therapy in patients infected with the Middle East respiratory syndrome coronavirus: an observational study. Int J Infect Dis 20:42-46. 10.1016/j.ijid.2013.12.003

Alfaraj SH, Al-Tawfiq JA, Altuwaijri TA, Alanazi M, Alzahrani N, and Memish ZA. 2018. Middle East respiratory syndrome coronavirus transmission among health care workers: Implication for infection control. Am J Infect Control 46:165-168. 10.1016/j.ajic.2017.08.010

Ali MA, Shehata MM, Gomaa MR, Kandeil A, El-Shesheny R, Kayed AS, El-Taweel AN, Atea M, Hassan N, Bagato O, Moatasim Y, Mahmoud SH, Kutkat O, Maatouq AM, Osman A, McKenzie PP, Webby RJ, and Kayali G. 2017. Systematic, active surveillance for Middle East respiratory syndrome coronavirus in camels in Egypt. Emerg Microbes Infect 6:e1. 10.1038/emi.2016.130

Alshukairi AN, Zheng J, Zhao J, Nehdi A, Baharoon SA, Layqah L, Bokhari A, Al Johani SM, Samman N, Boudjelal M, Ten Eyck P, Al-Mozaini MA, Zhao J, Perlman S, and Alagaili AN. 2018. High Prevalence of MERS-CoV Infection in Camel Workers in Saudi Arabia. MBio 9. 10.1128/mBio.01985-18

Arabi YM, Arifi AA, Balkhy HH, Najm H, Aldawood AS, Ghabashi A, Hawa H, Alothman A, Khaldi A, and Al Raiy B. 2014. Clinical course and outcomes of critically ill patients with Middle East respiratory syndrome coronavirus infection. Ann Intern Med 160:389-397. 10.7326/M13-2486

Aronin SI, and Sadigh M. 2004. Severe acute respiratory syndrome. Conn Med 68:207-215.

Arwady MA, Alraddadi B, Basler C, Azhar EI, Abuelzein E, Sindy AI, Sadiq BM, Althaqafi AO, Shabouni O, Banjar A, Haynes LM, Gerber SI, Feikin DR, and Madani TA. 2016. Middle East Respiratory Syndrome Coronavirus Transmission in Extended Family, Saudi Arabia, 2014. Emerg Infect Dis 22:1395-1402. 10.3201/eid2208.152015

Azhar EI, Hashem AM, El-Kafrawy SA, Sohrab SS, Aburizaiza AS, Farraj SA, Hassan AM, Al-Saeed MS, Jamjoom GA, and Madani TA. 2014. Detection of the Middle East respiratory syndrome coronavirus genome in an air sample originating from a camel barn owned by an infected patient. MBio 5:e01450-01414. 10.1128/mBio.01450-14

Bayrakdar F, Altas AB, Korukluoglu G, and Topal S. 2015. [Molecular diagnosis and phylogenetic analysis of the first MERS case in Turkey]. Mikrobiyol Bul 49:414-422.

CDC. 2016. Coronavirus I Home I CDC. (2016). gov. Retrieved 26 December 2016, from https://wwwcdcgov/coronavirus/.

Chan RW, Hemida MG, Kayali G, Chu DK, Poon LL, Alnaeem A, Ali MA, Tao KP, Ng HY, Chan MC, Guan Y, Nicholls JM, and Peiris JS. 2014. Tropism and replication of Middle East respiratory syndrome coronavirus from dromedary camels in the human respiratory tract: an in-vitro and exvivo study. Lancet Respir Med 2:813-822. 10.1016/S2213-2600(14)70158-4

Chan SM, Damdinjav B, Perera RA, Chu DK, Khishgee B, Enkhbold B, Poon LL, and Peiris M. 2015. Absence of MERS-Coronavirus in Bactrian Camels, Southern Mongolia, November 2014. Emerg Infect Dis 21:1269-1271. 10.3201/eid2107.150178

Peer] reviewing PDF | (2018:12:33627:2:2:NEW 21 Jun 2019) 
480 Choi S, Jung E, Choi BY, Hur YJ, and Ki M. 2017. High reproduction number of Middle East respiratory

481

482

483

484

485

486

487

488

489

490

491

492

493

494

495

496

497

498

499

500

501

502

503

504

505

506

507

508

509

510

511

512

513

514

515

516

517

518 syndrome coronavirus in nosocomial outbreaks: mathematical modelling in Saudi Arabia and South Korea. J Hosp Infect. 10.1016/j.jhin.2017.09.017

Chu DKW, Hui KPY, Perera R, Miguel E, Niemeyer D, Zhao J, Channappanavar R, Dudas G, Oladipo JO, Traore A, Fassi-Fihri O, Ali A, Demissie GF, Muth D, Chan MCW, Nicholls JM, Meyerholz DK, Kuranga SA, Mamo G, Zhou Z, So RTY, Hemida MG, Webby RJ, Roger F, Rambaut A, Poon LLM, Perlman S, Drosten C, Chevalier V, and Peiris M. 2018. MERS coronaviruses from camels in Africa exhibit region-dependent genetic diversity. Proc Natl Acad Sci U S A. 10.1073/pnas.1718769115

Corman VM, Jores J, Meyer B, Younan M, Liljander A, Said MY, Gluecks I, Lattwein E, Bosch BJ, Drexler JF, Bornstein S, Drosten C, and Muller MA. 2014. Antibodies against MERS coronavirus in dromedary camels, Kenya, 1992-2013. Emerg Infect Dis 20:1319-1322. 10.3201/eid2008.140596

Crameri G, Durr PA, Barr J, Yu M, Graham K, Williams OJ, Kayali G, Smith D, Peiris M, Mackenzie JS, and Wang LF. 2015. Absence of MERS-CoV antibodies in feral camels in Australia: Implications for the pathogen's origin and spread. One Health 1:76-82. 10.1016/j.onehlt.2015.10.003

Crameri G, Durr PA, Klein R, Foord A, Yu M, Riddell S, Haining J, Johnson D, Hemida MG, Barr J, Peiris M, Middleton D, and Wang LF. 2016. Experimental Infection and Response to Rechallenge of Alpacas with Middle East Respiratory Syndrome Coronavirus. Emerg Infect Dis 22:1071-1074. 10.3201/eid2206.160007

Darling ND, Poss DE, Schoelen MP, Metcalf-Kelly M, Hill SE, and Harris S. 2017. Retrospective, epidemiological cluster analysis of the Middle East respiratory syndrome coronavirus (MERSCoV) epidemic using open source data. Epidemiol Infect 145:3106-3114. 10.1017/S0950268817002345

David D, Rotenberg D, Khinich E, Erster O, Bardenstein S, van Straten M, Okba NMA, Raj SV, Haagmans BL, Miculitzki M, and Davidson I. 2018. Middle East respiratory syndrome coronavirus specific antibodies in naturally exposed Israeli llamas, alpacas and camels. One Health 5:65-68. 10.1016/j.onehlt.2018.05.002

de Wilde AH, Raj VS, Oudshoorn D, Bestebroer TM, van Nieuwkoop S, Limpens RW, Posthuma CC, van der Meer Y, Barcena M, Haagmans BL, Snijder EJ, and van den Hoogen BG. 2013. MERScoronavirus replication induces severe in vitro cytopathology and is strongly inhibited by cyclosporin A or interferon-alpha treatment. J Gen Virol 94:1749-1760. 10.1099/vir.0.052910-0

de Wit E, Prescott J, Baseler L, Bushmaker T, Thomas T, Lackemeyer MG, Martellaro C, Milne-Price S, Haddock E, Haagmans BL, Feldmann H, and Munster VJ. 2013. The Middle East respiratory syndrome coronavirus (MERS-CoV) does not replicate in Syrian hamsters. PLoS One 8:e69127. 10.1371/journal.pone.0069127

Destoumieux-Garzon D, Mavingui P, Boetsch G, Boissier J, Darriet F, Duboz P, Fritsch C, Giraudoux P, Le Roux F, Morand S, Paillard C, Pontier D, Sueur C, and Voituron Y. 2018. The One Health Concept: 10 Years Old and a Long Road Ahead. Front Vet Sci 5:14. 10.3389/fvets.2018.00014

Drosten C, Meyer B, Muller MA, Corman VM, Al-Masri M, Hossain R, Madani H, Sieberg A, Bosch BJ, Lattwein E, Alhakeem RF, Assiri AM, Hajomar W, Albarrak AM, Al-Tawfiq JA, Zumla AI, and 
519

520

521

522

523

524

525

526

527

528

529

530

531

532

533

534

535

536

537

538

539

540

541

542

543

544

545

546

547

548

549

550

551

552

553

554

555
Memish ZA. 2014. Transmission of MERS-coronavirus in household contacts. N Engl J Med 371:828-835. 10.1056/NEJMoa1405858

FAO. 2016. Understanding MERS-CoV at the animal-human interface. Technical meeting, 21-22 January 2016, Rome, Italy.

Farag EA, Reusken CB, Haagmans BL, Mohran KA, Stalin Raj V, Pas SD, Voermans J, Smits SL, Godeke GJ, Al-Hajri MM, Alhajri FH, Al-Romaihi HE, Ghobashy H, El-Maghraby MM, El-Sayed AM, Al Thani MH, Al-Marri S, and Koopmans MP. 2015. High proportion of MERS-CoV shedding dromedaries at slaughterhouse with a potential epidemiological link to human cases, Qatar 2014. Infect Ecol Epidemiol 5:28305. 10.3402/iee.v5.28305

Gao H, Yao H, Yang S, and Li L. 2016. From SARS to MERS: evidence and speculation. Front Med 10:377382. 10.1007/s11684-016-0466-7

Garcell HG, Garcia EG, Pueyo PV, Martin IR, Arias AV, and Alfonso Serrano RN. 2016. Outbreaks of brucellosis related to the consumption of unpasteurized camel milk. J Infect Public Health 9:523527. 10.1016/j.jiph.2015.12.006

Han HJ, Liu JW, Yu H, and Yu XJ. 2018. Neutralizing Monoclonal Antibodies as Promising Therapeutics against Middle East Respiratory Syndrome Coronavirus Infection. Viruses 10. 10.3390/v10120680

He L, Tai W, Li J, Chen Y, Gao Y, Li J, Sun S, Zhou Y, Du L, and Zhao G. 2019. Enhanced Ability of Oligomeric Nanobodies Targeting MERS Coronavirus Receptor-Binding Domain. Viruses 11. 10.3390/v11020166

Hemida MG, Al-Naeem A, Perera RA, Chin AW, Poon LL, and Peiris M. 2015. Lack of middle East respiratory syndrome coronavirus transmission from infected camels. Emerg Infect Dis 21:699-701. 10.3201/eid2104.141949

Hemida MG, Alnaeem A, Chu DK, Perera RA, Chan SM, Almathen F, Yau E, Ng BC, Webby RJ, Poon LL, and Peiris M. 2017a. Longitudinal study of Middle East Respiratory Syndrome coronavirus infection in dromedary camel herds in Saudi Arabia, 2014-2015. Emerg Microbes Infect 6:e56. 10.1038/emi.2017.44

Hemida MG, Chu DK, Poon LL, Perera RA, Alhammadi MA, Ng HY, Siu LY, Guan Y, Alnaeem A, and Peiris M. 2014. MERS coronavirus in dromedary camel herd, Saudi Arabia. Emerg Infect Dis 20:1231-1234. 10.3201/eid2007.140571

Hemida MG, Elmoslemany A, Al-Hizab F, Alnaeem A, Almathen F, Faye B, Chu DK, Perera RA, and Peiris M. 2017b. Dromedary Camels and the Transmission of Middle East Respiratory Syndrome Coronavirus (MERS-CoV). Transbound Emerg Dis 64:344-353. 10.1111/tbed.12401

Hijawi B, Abdallat M, Sayaydeh A, Alqasrawi S, Haddadin A, Jaarour N, Alsheikh S, and Alsanouri T. 2013. Novel coronavirus infections in Jordan, April 2012: epidemiological findings from a retrospective investigation. East Mediterr Health J 19 Suppl 1:S12-18.

Hofer U. 2013. Viral evolution: fooling the coronavirus proofreading machinery. Nat Rev Microbiol 11:662663. 10.1038/nrmicro3125

Peer) reviewing PDF | (2018:12:33627:2:2:NEW 21 Jun 2019) 
556 Hong KH, Choi JP, Hong SH, Lee J, Kwon JS, Kim SM, Park SY, Rhee JY, Kim BN, Choi HJ, Shin EC, Pai

557

558

559

560

561

562

563

564

565

566

567

568

569

570

571

572

573

574

575

576

577

578

579

580

581

582

583

584

585

586

587

588

589

590

591

592

593

594

H, Park SH, and Kim SH. 2017. Predictors of mortality in Middle East respiratory syndrome (MERS). Thorax. 10.1136/thoraxjnl-2016-209313

Kandeil A, Gomaa M, Shehata M, El-Taweel A, Kayed AE, Abiadh A, Jrijer J, Moatasim Y, Kutkat O, Bagato O, Mahmoud S, Mostafa A, El-Shesheny R, Perera RA, Ko RL, Hassan N, Elsokary B, Allal L, Saad A, Sobhy H, McKenzie PP, Webby RJ, Peiris M, Ali MA, and Kayali G. 2019. Middle East respiratory syndrome coronavirus infection in non-camelid domestic mammals. Emerg Microbes Infect 8:103-108. 10.1080/22221751.2018.1560235

Khalafalla AI, Lu X, Al-Mubarak AI, Dalab AH, Al-Busadah KA, and Erdman DD. 2015. MERS-CoV in Upper Respiratory Tract and Lungs of Dromedary Camels, Saudi Arabia, 2013-2014. Emerg Infect Dis 21:1153-1158. 10.3201/eid2107.150070

Kim K, Andrew SA, and Jung K. 2017. Public Health Network Structure and Collaboration Effectiveness during the 2015 MERS Outbreak in South Korea: An Institutional Collective Action Framework. Int J Environ Res Public Health 14. 10.3390/ijerph14091064

Kim SH, Chang SY, Sung M, Park JH, Bin Kim H, Lee H, Choi JP, Choi WS, and Min JY. 2016. Extensive Viable Middle East Respiratory Syndrome (MERS) Coronavirus Contamination in Air and Surrounding Environment in MERS Isolation Wards. Clin Infect Dis 63:363-369. 10.1093/cid/ciw239

Lau SK, and Chan JF. 2015. Coronaviruses: emerging and re-emerging pathogens in humans and animals. Virol J 12:209. 10.1186/s12985-015-0432-z

Lerner H, and Berg C. 2015. The concept of health in One Health and some practical implications for research and education: what is One Health? Infect Ecol Epidemiol 5:25300. 10.3402/iee.v5.25300

Makino S, Keck JG, Stohlman SA, and Lai MM. 1986. High-frequency RNA recombination of murine coronaviruses. J Virol 57:729-737.

Memish ZA, Al-Tawfiq JA, Alhakeem RF, Assiri A, Alharby KD, Almahallawi MS, and Alkhallawi M. 2015. Middle East respiratory syndrome coronavirus (MERS-CoV): A cluster analysis with implications for global management of suspected cases. Travel Med Infect Dis 13:311-314. 10.1016/j.tmaid.2015.06.012

Memish ZA, Mishra N, Olival KJ, Fagbo SF, Kapoor V, Epstein JH, Alhakeem R, Durosinloun A, Al Asmari M, Islam A, Kapoor A, Briese T, Daszak P, Al Rabeeah AA, and Lipkin WI. 2013. Middle East respiratory syndrome coronavirus in bats, Saudi Arabia. Emerg Infect Dis 19:1819-1823. 10.3201/eid1911.131172

Mollers M, Jonges M, Pas SD, van der Eijk AA, Dirksen K, Jansen C, Gelinck LB, Leyten EM, Thurkow I, Groeneveld PH, van Gageldonk-Lafeber AB, Koopmans MP, Timen A, and Netherlands MECOITot. 2015. Follow-up of Contacts of Middle East Respiratory Syndrome Coronavirus-Infected Returning Travelers, the Netherlands, 2014. Emerg Infect Dis 21:1667-1669. 10.3201/eid2109.150560

Monchatre-Leroy E, Boue F, Boucher JM, Renault C, Moutou F, Ar Gouilh M, and Umhang G. 2017. Identification of Alpha and Beta Coronavirus in Wildlife Species in France: Bats, Rodents, Rabbits, and Hedgehogs. Viruses 9. 10.3390/v9120364

Peer) reviewing PDF | (2018:12:33627:2:2:NEW 21 Jun 2019) 
595 Moon SY, and Son JS. 2017. Infectivity of an Asymptomatic Patient With Middle East Respiratory

596 Syndrome Coronavirus Infection. Clin Infect Dis 64:1457-1458. 10.1093/cid/cix170

597 Munster VJ, Adney DR, van Doremalen N, Brown VR, Miazgowicz KL, Milne-Price S, Bushmaker T,

598 Rosenke R, Scott D, Hawkinson A, de Wit E, Schountz T, and Bowen RA. 2016. Replication and

599

600

601 shedding of MERS-CoV in Jamaican fruit bats (Artibeus jamaicensis). Sci Rep 6:21878.

602

603

604 10.1038/srep21878

Niu P, Zhao G, Deng Y, Sun S, Wang W, Zhou Y, and Tan W. 2018. A novel human mAb (MERS-GD27) provides prophylactic and postexposure efficacy in MERS-CoV susceptible mice. Sci China Life Sci 61:1280-1282. 10.1007/s11427-018-9343-8

605

606

607

608

609

610

611

612

613

614

615

616

617

618

619

620

621

622

623

624

625

626

627

628

629

630

631

632

Peiris JS, Lai ST, Poon LL, Guan Y, Yam LY, Lim W, Nicholls J, Yee WK, Yan WW, Cheung MT, Cheng VC, Chan KH, Tsang DN, Yung RW, Ng TK, Yuen KY, and group Ss. 2003. Coronavirus as a possible cause of severe acute respiratory syndrome. Lancet 361:1319-1325.

Rajakaruna SJ, Liu WB, Ding YB, and Cao GW. 2017. Strategy and technology to prevent hospitalacquired infections: Lessons from SARS, Ebola, and MERS in Asia and West Africa. Mil Med Res 4:32. 10.1186/s40779-017-0142-5

Reeves T, Samy AM, and Peterson AT. 2015. MERS-CoV geography and ecology in the Middle East: analyses of reported camel exposures and a preliminary risk map. BMC Res Notes 8:801. 10.1186/s13104-015-1789-1

Reusken CB, Farag EA, Jonges M, Godeke GJ, El-Sayed AM, Pas SD, Raj VS, Mohran KA, Moussa HA, Ghobashy H, Alhajri F, Ibrahim AK, Bosch BJ, Pasha SK, Al-Romaihi HE, Al-Thani M, Al-Marri SA, AlHajri MM, Haagmans BL, and Koopmans MP. 2014. Middle East respiratory syndrome coronavirus (MERS-CoV) RNA and neutralising antibodies in milk collected according to local customs from dromedary camels, Qatar, April 2014. Euro Surveill 19.

Reusken CB, Schilp C, Raj VS, De Bruin E, Kohl RH, Farag EA, Haagmans BL, Al-Romaihi H, Le Grange F, Bosch BJ, and Koopmans MP. 2016. MERS-CoV Infection of Alpaca in a Region Where MERS$\mathrm{CoV}$ is Endemic. Emerg Infect Dis 22:1129-1131. 10.3201/eid2206.152113

Roess A, Carruth L, Lahm S, and Salman M. 2016. Camels, MERS-CoV, and other emerging infections in east Africa. Lancet Infect Dis 16:14-15. 10.1016/S1473-3099(15)00471-5

Rubio E, Martinez MJ, Gonzalo V, Barrachina J, Torner N, Martinez AI, Jane M, Vilella A, Del Rio A, Rodriguez-Valero N, Pinazo MJ, Munoz J, Soriano A, Trilla A, Vila J, and Marcos MA. 2018. Definitive diagnosis in suspected Middle East Respiratory Syndrome Coronavirus cases. J Travel Med 25. 10.1093/jtm/tax084

Totura AL, and Bavari S. 2019. Broad-spectrum coronavirus antiviral drug discovery. Expert Opin Drug Discov 14:397-412. 10.1080/17460441.2019.1581171

van Doremalen N, Bushmaker T, Karesh WB, and Munster VJ. 2014. Stability of Middle East respiratory syndrome coronavirus in milk. Emerg Infect Dis 20:1263-1264. 10.3201/eid2007.140500

van Doremalen N, Hijazeen ZS, Holloway P, Al Omari B, McDowell C, Adney D, Talafha HA, Guitian J, Steel J, Amarin N, Tibbo M, Abu-Basha E, Al-Majali AM, Munster VJ, and Richt JA. 2017. High 
633 Prevalence of Middle East Respiratory Coronavirus in Young Dromedary Camels in Jordan.

634 Vector Borne Zoonotic Dis 17:155-159. 10.1089/vbz.2016.2062

635

636

Vergara-Alert J, van den Brand JM, Widagdo W, Munoz Mt, Raj S, Schipper D, Solanes D, Cordon I,

637

Bensaid A, Haagmans BL, and Segales J. 2017. Livestock Susceptibility to Infection with Middle

638 East Respiratory Syndrome Coronavirus. Emerg Infect Dis 23:232-240. 10.3201/eid2302.161239

Wang M, Yan M, Xu H, Liang W, Kan B, Zheng B, Chen H, Zheng H, Xu Y, Zhang E, Wang H, Ye J, Li G,

639 Li M, Cui Z, Liu YF, Guo RT, Liu XN, Zhan LH, Zhou DH, Zhao A, Hai R, Yu D, Guan Y, and Xu J. 2005. SARS-CoV infection in a restaurant from palm civet. Emerg Infect Dis 11:1860-1865.

642

643 10.3201/eid1112.041293

WHO. 2018. Middle East Respiratory syndrome cornavirus (MERS-CoV).

644 http://wwwwhoint/emergencies/mers-cov/en/.

Widagdo W, Begeman L, Schipper D, Run PRV, Cunningham AA, Kley N, Reusken CB, Haagmans BL,

645

646 and van den Brand JMA. 2017. Tissue Distribution of the MERS-Coronavirus Receptor in Bats. Sci

647 Rep 7:1193. 10.1038/s41598-017-01290-6

Xiao S, Li Y, Sung M, Wei J, and Yang Z. 2018. A study of the probable transmission routes of MERS-CoV during the first hospital outbreak in the Republic of Korea. Indoor Air 28:51-63. 10.1111/ina.12430

650

651

652

653

654

655

656

657

658

659

660

661

662

663

Yu P, Xu Y, Deng W, Bao L, Huang L, Xu Y, Yao Y, and Qin C. 2017. Comparative pathology of rhesus macaque and common marmoset animal models with Middle East respiratory syndrome coronavirus. PLoS One 12:e0172093. 10.1371/journal.pone.0172093

Zaki AM, van Boheemen S, Bestebroer TM, Osterhaus AD, and Fouchier RA. 2012. Isolation of a novel coronavirus from a man with pneumonia in Saudi Arabia. N Engl J Med 367:1814-1820. 10.1056/NEJMoa1211721

Zhao G, Jiang Y, Qiu H, Gao T, Zeng Y, Guo Y, Yu H, Li J, Kou Z, Du L, Tan W, Jiang S, Sun S, and Zhou Y. 2015. Multi-Organ Damage in Human Dipeptidyl Peptidase 4 Transgenic Mice Infected with Middle East Respiratory Syndrome-Coronavirus. PLoS One 10:e0145561. 10.1371/journal.pone.0145561

Zhou J, Li C, Zhao G, Chu H, Wang D, Yan HH, Poon VK, Wen L, Wong BH, Zhao X, Chiu MC, Yang D, Wang Y, Au-Yeung RKH, Chan IH, Sun S, Chan JF, To KK, Memish ZA, Corman VM, Drosten C, Hung IF, Zhou Y, Leung SY, and Yuen KY. 2017. Human intestinal tract serves as an alternative

664 Zumla A, Azhar EI, Arabi Y, Alotaibi B, Rao M, McCloskey B, Petersen E, and Maeurer M. 2015. Host-

665

666 directed therapies for improving poor treatment outcomes associated with the middle east respiratory syndrome coronavirus infections. Int J Infect Dis 40:71-74. 10.1016/j.ijid.2015.09.005

667 


\section{Figure 1}

Current theories regarding the MERS-CoV/human/animal interaction

There might be an unknown reservoir in the transmission cycle of MERS-CoV. Bats play a role in the context of MERS-CoV transmission. The virus is transmitted to dromedary camels through an unknown mechanism. The dromedary camels act as amplifying hosts for the virus. MERS-CoV is transmitted from dromedary camels to humans through the respiratory aerosols and some other unknown mechanisms. The virus is then transmitted among the human population through respiratory routes. The human-to-human transmission has been confirmed. The human-to-camel transmission still needs further clarification. Question marks indicate the non-confirmed phenomenon.

\section{Unknown reservoir/s Other animal/s}

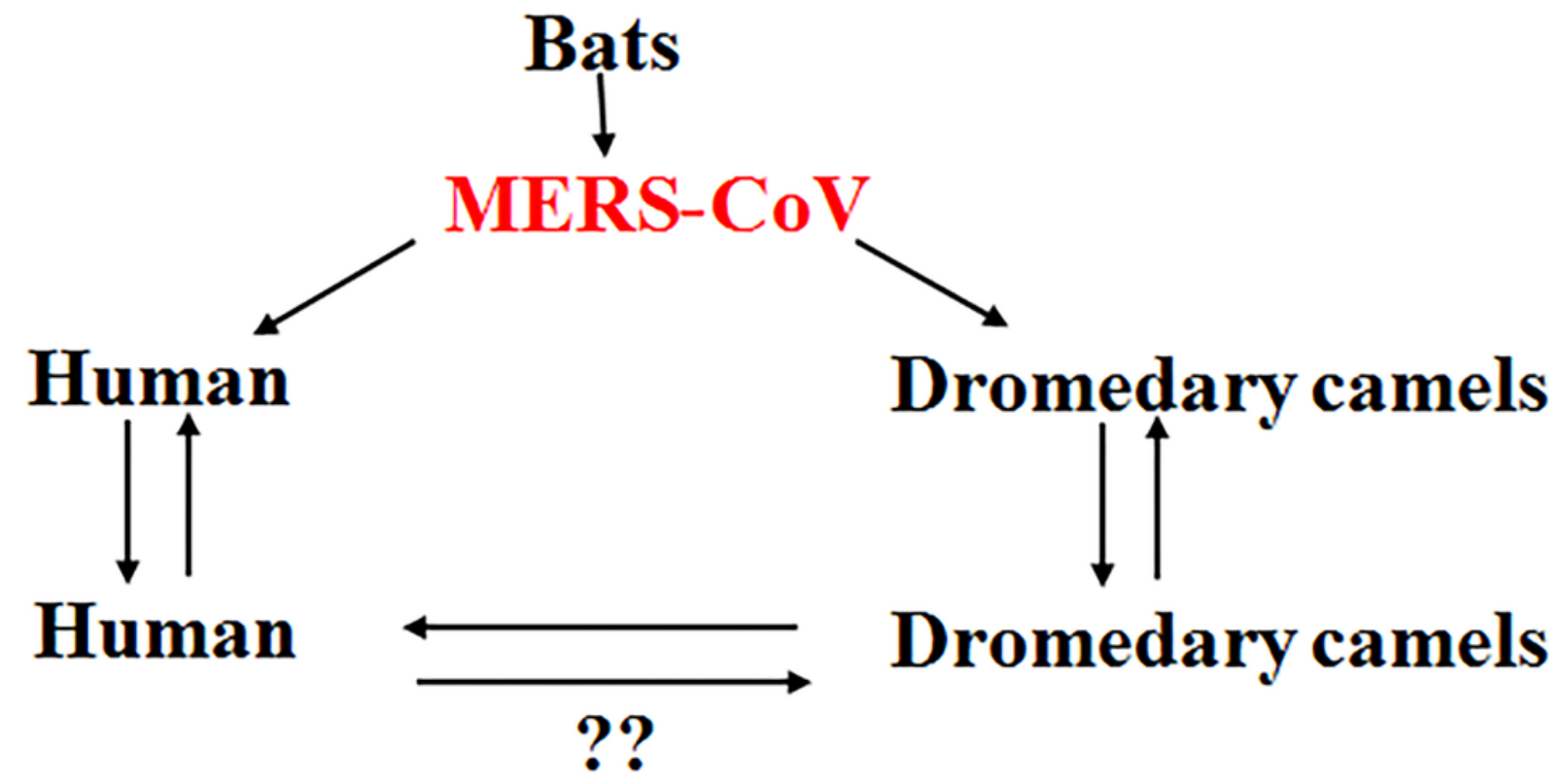


Figure 2

Figure 2

Some interventions based on the One Health-based to stop MERS-CoV outbreaks

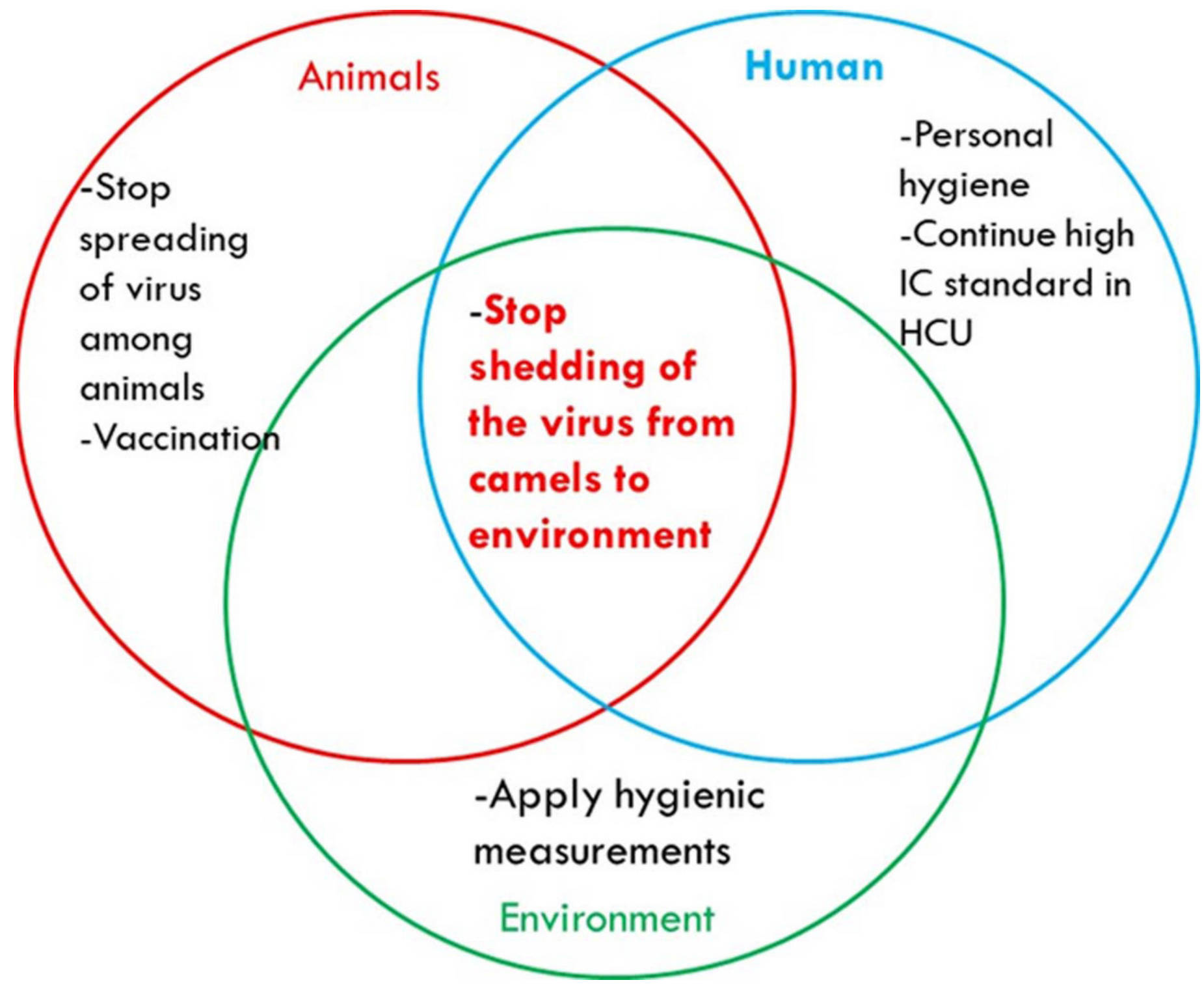

